\title{
Screening for Lung Cancer With Low-Dose Computed Tomography
}

\author{
Anthony B. Miller \\ Dalla Lana School of Public Health, University of Toronto, Toronto, Ontario, Canada \\ Disclosures of potential conflicts of interest may be found at the end of this article.
}

There is great enthusiasm for lung cancer screening using lowdose computed tomography (LDCT) following the publication of the results of the National Lung Screening Trial (NLST) [1]. This is understandable given the current low survival of patients diagnosed with symptomatic lung cancer and the very slow progress of improvements in treatment for the disease. Although there are several trials of lung screening using LDCT, the NLST is, and is likely to remain, the largest. One of the most remarkable features of the NLST is that benefit in terms of lower lung cancer mortality in the LDCT arm compared with the chest $x$-ray control arm began to accrue by the end of the first year after randomization and reached maximum benefit at 6 years. However, this early benefit has not been seen in three small European trials that have reported lung cancer mortality [2-4].

In addition to concern about whether it will be possible to replicate the NLST's results in the general population, it is important to recognize that there are a number of potential harms from LDCT screening (Table 1). They include hazard from the test itself, false reassurance from a false negative test, large numbers of false positive tests (low specificity), and complications from the investigation of false positive tests. In addition, there is earlier experience of "life with cancer" from true positive detection without extension of lifespan (lead time and overdiagnosis) with complications of unnecessarily early therapy and costs of unnecessary screening, diagnostic tests, and treatment. All of these costs represent a financial investment that might be applied more effectively to other health maneuvers - "opportunity" costs. Some of these will now be considered further.

\section{Hazards From the Test}

LDCT involves substantially more radiation than is needed for chest $\mathrm{x}$-rays. Under circumstances where a patient is symptomatic and a CT is required for diagnosis, the risk from radiation will generally be accepted, providing the dose is kept as low as reasonably achievable (the ALARA principle). However, in a screening program, when it is expected that the majority of those screened will not be found to have the disease, and especially when the program for an individual continues for many years, the harm from radiation could become important in further increasing the risk for lung cancer. Given that these programs will be recruiting those with a history of heavy smoking, there may be more harm to smokers than to non-smokers because we know there is a multiplicative interaction be- tween smoking and radiation in the induction of lung cancer. Further, repeat (diagnostic) CT will add to the radiation dose in those who have a positive test. The LDCT screening trials have not been followed long enough to evaluate such an effect, and now that follow-up of the NLST has ceased, such an evaluation may never be possible by direct evidence. However, in one microsimulation model based on data from the NLST, screening was beneficial but had a higher cost per quality-adjusted lifeyear when the model included radiation-induced lung cancers [5].

\section{Lack of Specificity from LDCT Screening}

Of the 74,477 screening procedures performed in the NLST, $17,497(23 \%)$ of the screens were false positive, for a specificity of $77 \%$ [1]. This is low for a screening test. Some of these positive screens were not considered to require diagnostic follow-up; however, 12,108 (16\%) were, of which 9,781 (13\%) patients underwent clinical testing and 9,597 (13\%) received further imaging.

Among those required to have diagnostic follow-up who were not found to have lung cancer, 227 had bronchoscopy and 66 underwent needle biopsy. One patient had a complication deemed major as a result of the testing, and 11 patients died within 60 days.

We must bear in mind that, even among those found to have lung cancer as a result of the screening, case detection is not equivalent to benefit. The detected cancer may not be curable, and its natural history may not be modified by available treatment (in NLST, 80\% of the expected lung cancer deaths still occurred), whereas cancer detected by screening may never have presented clinically in the patient's lifetime, which is what we term overdiagnosis.

\section{Overdiagnosis in NLST}

There were some important differences in the stage distribution of lung cancers detected at the three screening rounds of the NLST [1]. At each round, an excess of stage IA lung cancers was detected in the LDCT arm, totaling 336 versus 112 detected in the chest $\mathrm{x}$-ray arm, and suggesting substantial overdiagnosis. However, this excess was partially offset by a reduction in stage IV lung cancers detected in the LDCT arm at the second and third repeat screens (although the numbers were identical at the prevalence screen), totaling 106 and 143, respectively. Nevertheless, these differences were reflected in an excess of lung cancers ascertained in the LDCT group at the end of the re- 
Table 1. Summary of hazards from low-dose CT screening for lung cancer

\begin{tabular}{|c|c|}
\hline Hazard & Consequence \\
\hline \multirow[t]{2}{*}{$\begin{array}{l}\text { False reassurance from a } \\
\text { negative test }\end{array}$} & $\begin{array}{l}\text { - Subject ignores warning symptoms } \\
\text { in the belief he or she is free of } \\
\text { cancer }\end{array}$ \\
\hline & $\begin{array}{l}\text { - Subject persists with smoking in } \\
\text { the belief that he or she is at low risk } \\
\text { for cancer }\end{array}$ \\
\hline \multirow[t]{2}{*}{ Risk from radiation } & - Future increase in lung cancer \\
\hline & - Cardiac damage \\
\hline \multirow[t]{4}{*}{ Low specificity of the test } & - False positive screening tests \\
\hline & - Unnecessary diagnostic testing \\
\hline & - Unnecessary lung biopsies \\
\hline & $\begin{array}{l}\text { - Morbidity and mortality from } \\
\text { thoracic surgery }\end{array}$ \\
\hline \multirow{2}{*}{$\begin{array}{l}\text { Overdiagnosis of lung } \\
\text { cancer }\end{array}$} & - Living with cancer unnecessarily \\
\hline & $\begin{array}{l}\text { - Unnecessary lung cancer } \\
\text { resections }\end{array}$ \\
\hline Opportunity costs & $\begin{array}{l}\text { - Funds used for screening not } \\
\text { available for other aspects of cancer } \\
\text { control (e.g., smoking cessation } \\
\text { programs) }\end{array}$ \\
\hline
\end{tabular}

ported follow-up, 1,060 versus 941, respectively, an excess of 119 , representing $16.5 \%$ of the screen-detected cancers in the LDCT arm. This is a fair approximation of the extent of overdiagnosis in NLST, as shown by of Figure $1 \mathrm{~A}$ in the report [1]. The excess in numbers of lung cancer cases in the LDCT arm reached a maximum at the end of the period of screening. There is then a catch-up in cases diagnosed in the chest x-ray arm until about year 6 , after which the difference between the arms is relatively stable.

The comparison in NLST is between LDCT and chest $\mathrm{x}$ ray screening, and overdiagnosis was associated with chest $x$-ray screening in the Mayo Lung trial [6]. In the Prostate, Lung, Colorectal, and Ovarian (PLCO) screening trial, which evaluated chest $\mathrm{x}$-ray screening versus usual care, there was an indication of slight (nonsignificant) overdiagnosis [7]. Thus, throughout the period of follow-up, there was an excess of positive cases in the screening arm compared with the usual care arm. At 9 years of follow-up, there was an excess of 36 cases, more than $10 \%$ of the screen-detected cases. Adjusting the $16.5 \%$ overdiagnosis in NLST by this excess in PLCO suggests that LDCT results in overdiagnosis in more than one fourth of the cases compared with no screening, a not inconsiderable disadvantage of such screening.

Unfortunately, although we have some clues (e.g., those diagnosed with a broncho-alveolar carcinoma seem more likely to be overdiagnosed than those with other types of nonsmall cell lung cancer or small cell lung cancer), we cannot determine which patient is likely to be, or has been, overdiagnosed. This is why we must bear in mind the popularity paradox [8], "The greater the harm through overdiagnosis and overtreatment from screening, the more people there are who believe they owe their health, or even their life, to the programme."

\section{Opportunity Costs}

We cannot afford to do everything. The resources used for LDCT screening could reduce those available for the diagnosis and management of symptomatic patients, whereas new funds required for a screening program could divert funds from primary prevention, especially smoking cessation in current smokers. Smoking cessation is more costeffective than LDCT screening [5, 9], although cessation programs will not have an impact on those who have already given up smoking yet retain the lung cancer risk they had acquired up to the time of cessation. Therefore, ethical issues must be considered.

\section{Ethical Principles Related to Screening}

Screening is a public health process. Screening is initiated by the institutions doing the screening, not offered in response to a felt need by those would undergo screening. It is ethically imperative that those offering screening be able to guarantee those who agree to accept screening that they will receive more benefit than harm. So far, we do not have confirmation that this is so for LDCT screening, although mathematical modeling suggests that screening will be cost-effective, provided the program incorporates major efforts to promote smoking cessation in continuing smokers $[5,9]$.

\section{Major Residual Concerns}

It is not clear whether annual LDCT screening is affordable or whether it is necessary for maximum benefit; that is, we do not know how frequently LDCT has to be repeated to maintain a benefit, whether it is annually or every 2 years or every 5 years. However, we do know that relaxing the smoking recruitment criteria used in the NLST would increase the cost of screening considerably without an increase in benefit by increasing the opportunity costs. Indeed, some modeling is ongoing to determine whether stricter recruitment criteria, such as a more restricted (or older) age range, and a previous history of greater smoking, such as 40 pack-years, should be used rather than the NLST criteria.

\section{CoNCLUSION}

There are still a number of unanswered questions before we can be satisfied that an agreed policy of screening for lung cancer with LDCT can be introduced. This was also the conclusion of an American multisociety collaborative initiative [10]. In considering lung screening, we should not ignore the difficulties other screening programs have encountered, not the least of which is recruiting a sufficient proportion of participants for the at-risk group to obtain an impact at the population level. Further, the failure of other trials to replicate the early benefit seen in NLST is troubling and suggests that the type of respondents recruited may have implications on the results achieved.

\section{DisCLOSURES}

The author indicated no financial relationships. 


\section{REFERENCES}

1. National Lung Screening Trial Research Team. Reduced lung-cancer mortality with low-dose computed tomographic screening. N Engl J Med 2011;365:395409.

2. Infante $M$, Cavuto $S$, Lutman FR et al. DANTE Study Group. A randomized study of lung cancer screening with spiral computed tomography: Three-year results from the DANTE trial Am J Respir Crit Care Med 2009;180:445-453.

3. Saghir Z, Dirksen A, Ashraf H et al. CT screening for lung cancer brings forward early disease: The randomised Danish Lung Cancer Screening Trial: Status after five annual screening rounds with lowdose CT. Thorax 2012;67:296-301.
4. Pastorino U, Rossi M, Rosato V et al. Annual or biennial CT screening versus observation in heavy smokers: 5-year results of the MILD trial. Eur J Cancer Prev 2012;21:308-315.

5. McMahon PM, Kong CY, Bouzan C et al. Costeffectiveness of computed tomography screening for lung cancer in the United States. J Thorac Oncol 2011;6:1841-1848.

6. Marcus PM, Bergstralh EJ, Zweig MH et al. Extended lung cancer incidence follow-up in the Mayo Lung Project and overdiagnosis. J Natl Cancer Inst 2006;98:748-756.

7. Oken MM, Hocking WG, Kvale PA et al. Screening by chest radiograph and lung cancer mortality: The Prostate, Lung, Colorectal, and Ovarian (PLCO) randomized trial JAMA 2011;306: 1865-1873.

8. Raffle $A E$, Gray JAM. Screening: Evidence and Practice. Oxford, UK: Oxford University Press, 2007 366.

9. Evans WK, Wolfson MC. Computed tomography screening for lung cancer without a smoking cessation program - not a cost-effective idea. J Thorac Oncol 2011;6:1781-1783.

10. Bach PB, Mirkin JN, Oliver TK et al. Benefits and harms of CT screening for lung cancer. A systematic review. JAMA 2012;307:2418-2429.

EDITOR'S NOTE: For a comprehensive overview of the use of low-dose computed tomography for lung cancer screening, including strategies for the implementation of lung cancer screening programs, please see "Moving Beyond the National Lung Screening Trial: Discussion Strategies for Implementation of Lung Cancer Screening Programs," by Bernard H.L. Goulart and Scott D. Ramsey, on pages $941-946$ of this issue. 